\title{
Analysis of Geological Materials Containing Uranium using Laser-Induced Breakdown Spectroscopy (LIBS)
}

\author{
James E. Barefield $\mathrm{II}^{1}$, Elizabeth J. Judge ${ }^{1}$, Keri R. Campbell ${ }^{1}$, James P. Colgan ${ }^{3}$, David P. Kilcrease, \\ Heather M. Johns ${ }^{3}$, Roger C. Wiens ${ }^{4}$, Rhonda E. McInroy ${ }^{2}$, Ronald K. Martinez ${ }^{1}$, Samuel M. Clegg ${ }^{2}$ \\ ${ }^{1}$ Chemical Diagnostics and Engineering \\ ${ }^{2}$ Physical Chemistry and Applied Spectroscopy \\ ${ }^{3}$ Physics and Chemistry of Materials \\ ${ }^{4}$ Space and Remote Sensing \\ Los Alamos National Laboratory, Los Alamos, New Mexico 87545
}

\begin{abstract}
Laser induced breakdown spectroscopy (LIBS) is a rapid atomic emission spectroscopy technique that can be configured for a variety of applications including space, forensics, and industry. LIBS can also be configured for stand-off distances or in-situ, under vacuum, high pressure, atmospheric or different gas environments, and with different resolving-power spectrometers. The detection of uranium in a complex geological matrix under different measurement schemes is explored in this paper. Although many investigations have been completed in an attempt to detect and quantify uranium in different matrices at in-situ and standoff distances, this work detects and quantifies uranium in a complex matrix under Martian and ambient air conditions. Investigation of uranium detection using a low resolving-power LIBS system at stand-off distances $(1.6 \mathrm{~m})$ is also reported. The results are compared to an in-situ LIBS system with medium resolving power and under ambient air conditions. Uranium has many thousands of emission lines in the 200-800nm spectral region. In the presence of other matrix elements and at lower concentrations, the limit of detection of uranium is significantly reduced. The two measurement methods (low and high resolving-power spectrometers) are compared for limit of detection (LOD). Of the twentyone potential diagnostic uranium emission lines, seven $(409,424,434,435,436,591$, and $682 \mathrm{~nm})$ have been used to determine the LOD for pitchblende in a dunite matrix using the ChemCam test bed LIBS system. The LOD values determined for uranium transitions in air are $409.013 \mathrm{~nm}$ (24700 ppm), 424.167 $\mathrm{nm}$ (23780 ppm), $434.169 \mathrm{~nm}$ (24390 ppm), $435.574 \mathrm{~nm}$ (35880 ppm), $436.205 \mathrm{~nm}$ (19340 ppm), $591.539 \mathrm{~nm}(47310 \mathrm{ppm})$, and $682.692 \mathrm{~nm}(18580 \mathrm{ppm})$. The corresponding LOD values determined for uranium transitions in 7 torr $\mathrm{CO}_{2}$ are $424.167 \mathrm{~nm}(25760 \mathrm{ppm}), 434.169 \mathrm{~nm}(40800 \mathrm{ppm}), 436.205 \mathrm{~nm}$ (32050 ppm), $591.539 \mathrm{~nm}(15,340 \mathrm{ppm})$, and $682.692 \mathrm{~nm}(29080 \mathrm{ppm})$. The LOD values determine for uranium emission lines using the medium resolving power $(10000 \lambda / \lambda)$ LIBS system for the dunite matrix in air are $409.013 \mathrm{~nm}$ (6120 ppm), $424.167 \mathrm{~nm}$ (5356 ppm), $434.169 \mathrm{~nm}$ (5693 ppm), $435.574 \mathrm{~nm}$ (6329 ppm), $436.205 \mathrm{~nm}(2142 \mathrm{ppm})$, and $682.692 \mathrm{~nm}(10741 \mathrm{ppm})$. The corresponding LOD values determined for uranium transitions in a $\mathrm{SiO}_{2}$ matrix are $409.013 \mathrm{~nm}(272 \mathrm{ppm}), 424.167 \mathrm{~nm}(268 \mathrm{ppm})$, $434.169 \mathrm{~nm}$ (402 ppm), $435.574 \mathrm{~nm}$ (1067 ppm), $436.205 \mathrm{~nm}$ (482 ppm), and $682.692 \mathrm{~nm}$ (720 ppm).The impact of spectral resolution, atmospheric conditions, matrix elements, and measurement distances on LOD are discussed. The measurements will assist one in selecting the proper system components based upon the application and the required analytical performance.
\end{abstract}

Keywords: Laser-induced breakdown spectroscopy, Uranium, geological analysis, limit of detection, spectrometer resolving power 


\section{Introduction}

Laser-induced breakdown spectroscopy (LIBS) has been used to analyze the elemental composition and concentration of many complex environmental samples. Elements from hydrogen to the heavy elements including actinides (uranium, plutonium, americium, neptunium, and thorium) have been investigated [119]. LIBS is a highly configurable technique based on the needs of the application. In-situ measurements can be readily replaced with stand-off measurements for hard to reach samples due to rough terrain or a hazardous area by changing laser beam launching and emission light collection optics. LIBS measurements under different atmospheric conditions such as under vacuum, gas pressure, or even other planetary atmospheres have also been achieved for applications on Mars or to reduce atmospheric interferences [19]. An important experimental parameter for performing a LIBS measurement is the resolving power of the spectrometer. This aspect of the system tends to be a balance between size restrictions and spectral assignment and sensitivity requirements. Low resolving power spectrometers typically have a 200-1000 nm spectral range and tend to be much smaller in size. However, sensitivity and/or accuracy in emission line assignment due to several emission lines being convoluted in a single spectral feature are the major downfall. High resolving power spectrometers tend to be much larger and have more narrow spectral regions but are capable of baseline separation of elemental isotopes [21]. Other novel techniques for the detection and analysis of uranium have been reported by Ko and Jovanovic [30]. In summary, the application, the chemical environment of the sample (matrix), and the quantitative or qualitative needs dictate the system requirements.

The focus of this work is to detect and quantify uranium and determine limit of detection (LOD) under different measurement schemes. The measurements will assist one in selecting the proper system components based upon the application and the required analytical performance. The detection and analysis of uranium in complex matrices has been the subject of many investigations since the work of Wachter and Cremers in 1987 [7]. In that investigation, LIBS was used to determine a LOD of $100 \mathrm{ppm}$ for uranium in a nitric acid solution. A review of the result from previous investigations to quantify uranium in a variety of matrices is shown in Table I. In summary, LIBS has been used to identify and provide semi-quantitative determinations of uranium in many different matrices at in situ and standoff distances. Most of the experimental results reported so far have been conducted under ambient air conditions. The primary challenge in using LIBS to accurately identify and assign atomic emission spectral signatures for uranium is to exploit the complex interplay between experimental parameters (delay time, gate width, laser power, atmospheric compositions including ambient air, $\mathrm{Ar}, \mathrm{He}, \mathrm{CO}_{2}$, etc.) and concentration to determine the lowest LOD for uranium in a matrix. For example, isolating an atomic emission line from neighboring transitions that have different concentration dependencies on integrated intensity is of high importance. Therefore, resolving power of dispersion devices (spectrometers) and signal to noise (background) ratios are extremely important instrument and experimental parameters.

Another important factor is the data analysis method used to determine LOD. Kim et. al. found that normalization to Ca I or Fe I using the same $356.659 \mathrm{~nm} \mathrm{U} \mathrm{I} \mathrm{line} \mathrm{resulted} \mathrm{in} \mathrm{limit} \mathrm{of} \mathrm{detections} \mathrm{of} 158$ or $186 \mathrm{ppm}$ respectively [12]. Others use mathematical techniques such as multi-component curve fitting and partial least squares to de-convolute the partially resolved peaks in the spectrum [13] This technique results in reported LOD of 17-51 ppm for U II 409 and 3-12 ppm for U I 682 with a NIST 1872 SRM, 
where the resolving power of the spectrometer was reported as 34,000 [13]. In this work, the limit of detection (LOD) is defined as $3.3 \sigma / \mathrm{m}$ where $\sigma$ is the standard deviation of the y-intercept of the calibration curve based on the regression analysis and $m$ is the linear slope of the calibration curve [22]. This approach is a better indicator of the LOD at low concentrations rather than averages derived at higher concentrations from the residual standard deviations. Other methods to calculate LOD are described by Lasue et. al [23].

Although many investigations have been completed in an attempt to detect and quantify uranium in different matrices at in situ and standoff distances, this is the first report of detecting and quantifying uranium in a complex matrix under Martian conditions. The ChemCam test bed LIBS system is used as the low resolving-power standoff LIBS system to identify and assign uranium atomic emission lines [20]. Several uranium atomic emission lines are measured and discussed to quantify uranium at a standoff distance of 1.6 meters. These results are compared to medium resolving power LIBS in-situ measurements under ambient air conditions. The roles that spectral resolution, environmental conditions (7 Torr $\mathrm{CO}_{2}$ versus air), and matrix elements play on LOD are also reported.

Table I A list of detection and analysis results of uranium in different matrices from previous work.

\begin{tabular}{|c|c|c|c|c|}
\hline Author & $\begin{array}{l}\text { Transition } \\
(\mathbf{n m})^{1}\end{array}$ & Matrix & Resolving Power & LOD (ppm) \\
\hline $\begin{array}{c}\text { Wachter/ } \\
\text { Cremers [7] }\end{array}$ & 409 II & Nitric Acid & 4,000 & 100 \\
\hline $\begin{array}{c}\text { Chinni/Cremers } \\
\text { [9] }\end{array}$ & 409 II & silica & 6,000 & $\begin{array}{c}2600(\text { in Situ }) / \\
5000(@ 30 \mathrm{~m})^{2}\end{array}$ \\
\hline \multirow[t]{4}{*}{ Sarkar [10] } & $263 \mathrm{II}$ & thoria & 2,000 & 37,000 \\
\hline & 367 II & thoria & 2,000 & 38,000 \\
\hline & $447 \mathrm{I}$ & thoria & 2,000 & $\mathrm{NR}^{3}$ \\
\hline & 454 II & thoria & 2,000 & 34,000 \\
\hline Jung [11] & $358 \mathrm{I}$ & silica & 40,000 & 150 \\
\hline $\operatorname{Kim}[12]$ & $356 \mathrm{I}$ & silica & 40,000 & 158 \\
\hline \multirow[t]{2}{*}{ Choi [13] } & $409 \mathrm{II}$ & $\begin{array}{l}\text { Pb-germanate } \\
\text { glass }\end{array}$ & 34,000 & $17-51$ \\
\hline & $682 \mathrm{I}$ & $\begin{array}{c}\text { Pb-germanate } \\
\text { glass }\end{array}$ & 34,000 & $3-12$ \\
\hline
\end{tabular}

\section{Experimental}

\section{Instrumentation}

Standoff LIBS System

A detailed description of the ChemCam test bed LIBS system used in this investigation has been previously described by Maurice, et. al. [24] and Wiens, et. al. [25], therefore only a brief description will be provided in this paper. The excitation source was a diode pumped Nd:KGW laser (designed and built by CNES and Thales Optronics, France) operating at $1067 \mathrm{~nm}$ producing pulses of $5 \mathrm{~ns}$ width at a repetition rate of $3 \mathrm{~Hz}$. The maximum output energy was $13 \pm 1 \mathrm{~mJ}$ per pulse. The output excitation energy 
from this laser was focused to a spot size of $300 \mu \mathrm{m}$ on target using a beam expander at a standoff distance of 1.6 meters. A Schmidt Cassegrain telescope was used to collect the emission from the plasma and transfer it to three spectrometers via a de-multiplexer. The spectrometers cover three spectral regions: 240.8-340.8 nm, 382.1-469.1 nm, and 473.1-905.6 nm with a resolving powers of 1930, 2125, and 1150 respectively [20,24]. Compared to traditional time-gated LIBS systems, this system is operated in a nongated mode with an integration time of approximately $3 \mathrm{~ms}$. Each time the laser is fired, the shutter on the spectrometers opens for $3 \mathrm{~ms}$ and spectral data is collected. A total of 50 laser shots are acquired and the average spectrum is reported. The samples used in this investigation were analyzed in a vacuum chamber containing 7 Torr $\mathrm{CO}_{2}$ gas to simulate the atmospheric conditions on Mars and in air under ambient conditions.

\section{In-Situ LIBS System}

A medium resolving power echelle spectrometer from LLA Instruments (ESA 4000) was also used to collect LIBS spectral data between 220-780 nanometers. The resolving power of the spectrometer system is approximately 10,000. The laser excitation source used in these experiments was a Big Sky Ultra 100 pulsed Nd:YAG laser operating at $1064 \mathrm{~nm}$ with $8 \mathrm{~ns}$ pulses and a variable attenuator producing pulse energies up to $100 \mathrm{~mJ}$ at $20 \mathrm{~Hz}$. A laser pulse energy of approximately $10 \mathrm{~mJ}$ at $10 \mathrm{~Hz}$ was used for all experiments. A $2.5 \mathrm{~cm}$ diameter plano-convex focusing lens with a focal length of approximately $10 \mathrm{~cm}$ was used to focus the laser pulses onto the sample, perpendicular to the surface of the sample. A broadband UV/VIS/NIR 1 meter fiber optic cable with an $400 \mu \mathrm{m}$ inner core diameter was used to collect the plasma light $20^{\circ}$ off normal, approximately $6.3 \mathrm{~cm}$ from the plasma-sample interface. The fiber optic cable collected the plasma emission and transferred the light to the spectrometer. Gate width and delay time used in these experiments were $1 \mu \mathrm{s}$ and $100 \mathrm{~ns}$, respectively. Each spectrum presented here is an average of 200 laser shots (individual spectra) rastered across the surface of the sample. LIBS spectral data were collected from this system in air under standard atmospheric conditions. A comparison of the two LIBS systems used in this study can be found in Table II.

Table II Comparison of LIBS systems used in this study.

\begin{tabular}{|c|c|c|}
\hline Specifications & ChemCam test bed LIBS System & In-Situ LIBS System \\
\hline Laser Power & $13 \pm 1 \mathrm{~mJ}$ & $10 \mathrm{~mJ}$ \\
\hline Repetition Rate & $3 \mathrm{~Hz}$ & $10 \mathrm{~Hz}$ \\
\hline Wavelength (nm) & 1067 & 1064 \\
\hline Pulse Width & $5 \mathrm{~ns}$ & $8 \mathrm{~ns}$ \\
\hline Configuration & Standoff, $1.6 \mathrm{~m}$ & in-situ \\
\hline $\begin{array}{l}\text { No. Laser Shots per } \\
\text { Spectrum }\end{array}$ & 50 & 200 \\
\hline Spectrometer & Czerny-Turner & Echelle \\
\hline $\begin{array}{l}\text { Spectral Resolving } \\
\text { Power }\end{array}$ & $1150-2125^{1}$ & 10,000 \\
\hline Spectral Region & $\begin{array}{c}240.8-340.8 \mathrm{~nm}, 382.1-469.1 \mathrm{~nm}, \\
473.1-905.6 \mathrm{~nm}\end{array}$ & $200-1000 \mathrm{~nm}$ \\
\hline Gate Delay & - & $100 \mathrm{~ns}$ \\
\hline Gate Width & $3 \mathrm{~ms}$ & $1 \mu \mathrm{s}$ \\
\hline Atmosphere & 7 torr $\mathrm{CO}_{2}$ & Standard atmospheric conditions \\
\hline
\end{tabular}




\section{Samples}

The samples analyzed in this work were certified reference powders ( $\sim 5$ grams) that were pressed at 5,000 psi for 5 minutes to form a pellet ( 1 inch diameter discs). The samples analyzed in this investigation include the following: (1) a BL-5 uranium ore containing $7 \mathrm{wt} . \%$ uranium as uraninite (Minerals and Metals Sector of Natural Resources Canada), (2) pitchblende in silica (102A-105A series, New Brunswick Laboratory, 0.01\%, 0.05\%, 0.1\%, 1\% uranium), (3) pitchblende in dunite $\left((\mathrm{MgFe})_{2} \mathrm{SiO}_{4}\right)(42 \mathrm{~A}$ 1-4 series, New Brunswick Laboratory, $0.5 \%, 1 \%, 2 \%, 4 \%$ uranium), and (4) a depleted uranium (DU) metal sample. The approximate chemical composition of the BL-5 uranium ore sample is shown in Table III where the remaining weight percent is oxygen. The BL-5 uranium ore sample is a complex mineralogical sample containing plagioclase feldspar, hematite, quartz, calcite, dolomite, chlorite, muscovite, and uraninite. The BL-5 uranium ore sample represents a very complex and challenging environmental sample. The standoff low-resolving-power ChemCam test bed LIBS system was used to measure the uranium ore samples (as described in (1) and (3) above). Sample (4), depleted uranium, was also measured with this LIBS system to verify uranium emission features in the low resolving power spectrum. The in-situ medium resolving power LIBS system was used to analyze the uranium ores $(1,3)$ and the pitchblende in silica (2).

Table III Chemical composition of the BL-5 uranium ore certified reference material

\begin{tabular}{llllllllllllllllllll}
\hline Elements & $\mathbf{S i}$ & $\mathbf{U}$ & $\mathbf{A l}$ & $\mathbf{F e}$ & $\mathbf{C a}$ & $\mathbf{N a}$ & $\mathbf{C}$ & $\mathbf{P b}$ & $\mathbf{M g}$ & $\mathbf{K}$ & $\mathbf{T i}$ & $\mathbf{S}$ & $\mathbf{V}$ & $\mathbf{P}$ & $\mathbf{M n}$ & $\mathbf{Z r}$ & $\mathbf{S r}$ & $\mathbf{C r}$ & $\mathbf{T h}$ \\
\hline Weight $\%$ & 22 & 7.1 & 6 & 6 & 4 & 4 & 2 & 2 & 1.5 & 0 & 0 & 0 & 0 & 0.1 & 0.1 & 0 & 0 & 0 & 0 \\
\hline
\end{tabular}

\section{Results and Discussion}

\section{Detection and Analysis of Uranium under Standoff Conditions: 7 Torr $\mathrm{CO}_{2}$ and Standard Atmospheric Conditions}

Depleted uranium metal was analyzed to verify uranium peak position in the uranium ore samples using the ChemCam test bed LIBS system. In Figure 1 A-D, the black trace shows the LIBS spectrum of depleted uranium (DU) metal while the red trace shows the LIBS spectrum of $7 \%$ uranium ore. The uranium emission lines in the DU spectrum are more intense, as expected, than in the $7 \%$ uranium ore spectrum. However, the assignment of uranium emission lines to the small spectral features observed in the $7 \%$ uranium ore are validated due to the presence of those same emission lines in the DU spectrum. The identified and assigned atomic emission transitions are listed in Table IV below based on several references including previous published results from Judge and Barefield, [15,19] the high resolution work of Palmer, Keller, and Engleman [26] and the NIST and Kurucz databases [27,28]. There are several atomic emission transitions that may be used to determine concentration dependence. 


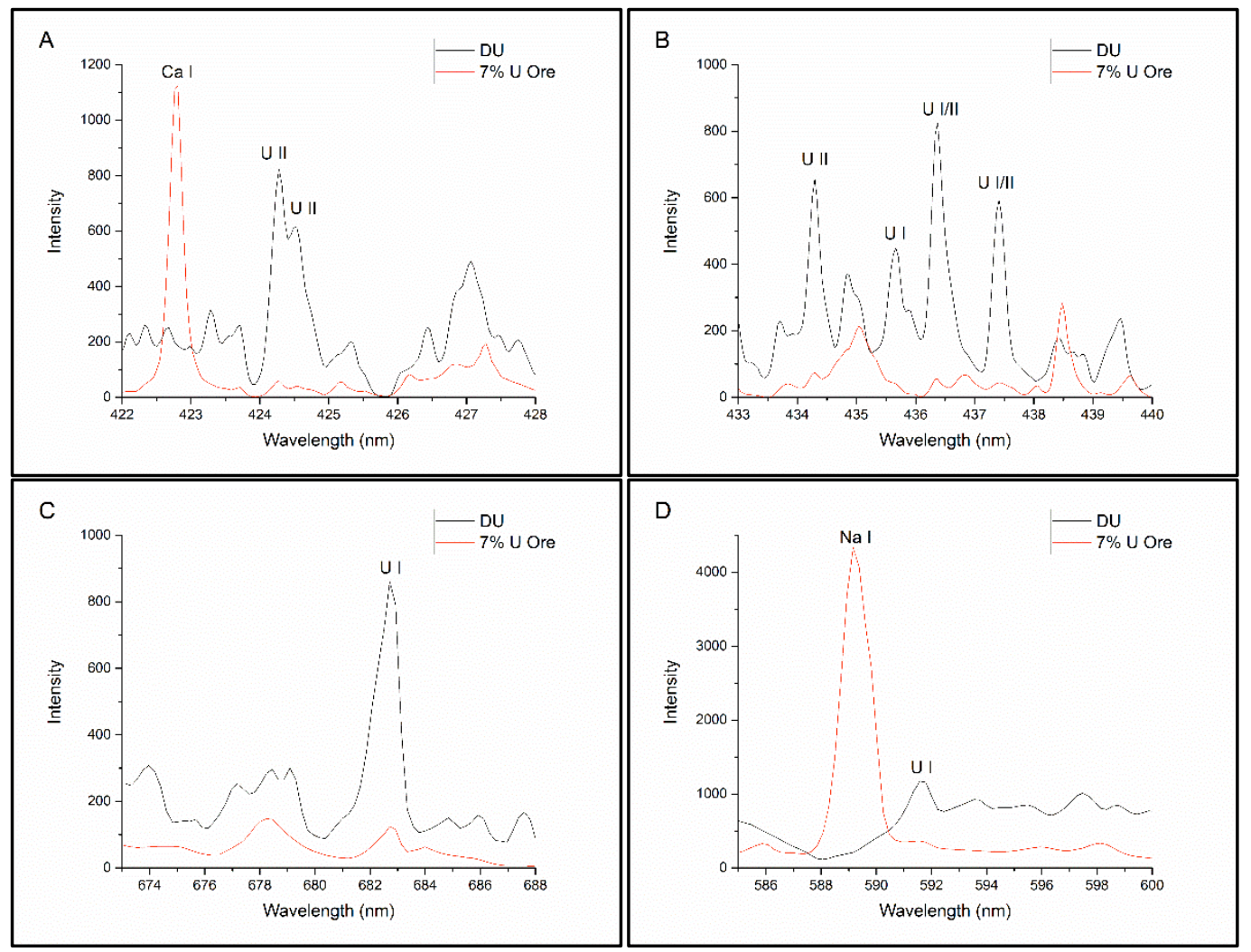

Fig. 1. Four spectral regions of the LIBS spectra of depleted uranium metal and 7\% uranium ore are shown as observed with the ChemCam test bed LIBS system, to assist in uranium line assignments: (A) 422-428 nm, (B) 433-440 nm, (C) 673-688 nm, and (D) $585-600 \mathrm{~nm}$.

The concentration dependence of uranium in an ore or dunite matrix $(7 \% \mathrm{U}$ in ore, $4 \%, 2 \%$, and $1 \% \mathrm{U}$ in dunite) were investigated for several uranium emission lines under 7 Torr $\mathrm{CO}_{2}$ and standard atmospheric conditions. Figure 2 A-D shows four spectral windows of interest for the detection of uranium under 7 Torr $\mathrm{CO}_{2}$ and at a standoff distance of $1.6 \mathrm{~m}:$ A) $422-427.5 \mathrm{~nm} \mathrm{B)} 433-440 \mathrm{~nm}$, C) 585-600 nm, and D) 673-688 nm. The 434, 436, and $437 \mathrm{~nm}$ emission lines in Figure 2B also show concentration dependence. The $591 \mathrm{~nm} \mathrm{U}$ I emission line in Figure 2C is adjacent to the sodium ( $\mathrm{Na} \mathrm{I})$ doublet at 588.99 and 589.59 $\mathrm{nm}$ which appear as a single peak due to the low resolving power of the spectrometer. However, peak intensity for several of the uranium emission lines can be seen to track with concentration. Figure 3A-C shows the comparison of three spectral regions of $4 \%$ and $7 \%$ uranium ore under standard atmospheric conditions and 7 Torr $\mathrm{CO}_{2}$. The $409 \mathrm{~nm}$ uranium emission line under 7 Torr $\mathrm{CO}_{2}$ shown in Figure $3 \mathrm{~A}$, could not be resolved from a neighboring peak whereas under standard atmospheric conditions, the 409 $\mathrm{nm}$ line is spectrally resolved. The $435 \mathrm{~nm}$ emission line appeared as a small shoulder on a broad spectral feature in the $\mathrm{CO}_{2}$ data as shown in Figure 3B. The interferences at low pressure ( $<10$ Torr) in $\mathrm{CO}_{2}$ is consistent with literature where the LIBS spectra tend to degrade under these conditions due to lack of plasma confinement [29].

Of the 21 uranium emission lines identified in Table IV, seven $(409,424,434,435,436,591$, and 682 $\mathrm{nm}$ ) were used to determine LODs at a standoff distance of 1.6 meters under standard atmospheric conditions and five transitions were used under Mars atmospheric conditions shown in Table V. With the ChemCam test bed LIBS system, the LOD values range from 15340-40800 ppm in $\mathrm{CO}_{2}$ and 18580-47310 ppm in air. In Figure 3, only the 4\% and 7\% uranium samples for air and $\mathrm{CO}_{2}$ are shown for spectral 
clarity. All four uranium samples as described in the experimental section were used to determine the LOD. The $\mathrm{R}^{2}$ for the calibration curves used to determine the LOD are shown in Table V. The uranium atomic emission transitions shown in Figures 2 and 3 are identified as small spectral features superimposed on large backgrounds that are a combination of noise and unresolved spectra. The integrated intensities of the uranium atomic emission transitions used in the determination of LODs are not completely separated from the background contribution. This leads to LODs that are not as accurate as would have been determined if the uranium transitions had been baseline resolved. However, the LOD values in a dunite matrix compare very well to the LOD values reported by Sarkar considering that both measurements were performed using low resolving power (1150- 2125 ChemCam and 2000 Sarkar) spectrometers [10].

\begin{tabular}{|c|c|}
\hline $\begin{array}{l}\text { Neutral (I) } \\
\text { or Ionic (II) }\end{array}$ & $\begin{array}{l}\text { Wavelength } \\
\text { (nm) }\end{array}$ \\
\hline U II & 409.013 \\
\hline U II & 417.159 \\
\hline U II & 424.167 \\
\hline U II & 424.437 \\
\hline $\mathbf{U} \mathbf{I}$ & 424.626 \\
\hline U II & 434.169 \\
\hline $\mathbf{U} \mathbf{I}$ & $\begin{array}{l}435.455 \\
435.574\end{array}$ \\
\hline $\mathbf{U} \mathbf{I} / \mathbf{I I}$ & $\begin{array}{l}436.221 \\
436.293\end{array}$ \\
\hline $\mathbf{U} \mathbf{I} / \mathbf{I I}$ & $\begin{array}{l}437.176, \\
437.275 \\
437.341\end{array}$ \\
\hline U I/II & $\begin{array}{l}454.362 \\
454.560\end{array}$ \\
\hline $\mathbf{U} \mathbf{I}$ & 462.028 \\
\hline U II & 462.707 \\
\hline $\mathbf{U} \mathbf{I}$ & 463.162 \\
\hline $\mathbf{U} \mathbf{I}$ & 591.539 \\
\hline $\mathbf{U} \mathbf{I}$ & 639.542 \\
\hline $\mathbf{U} \mathbf{I}$ & 682.692 \\
\hline
\end{tabular}
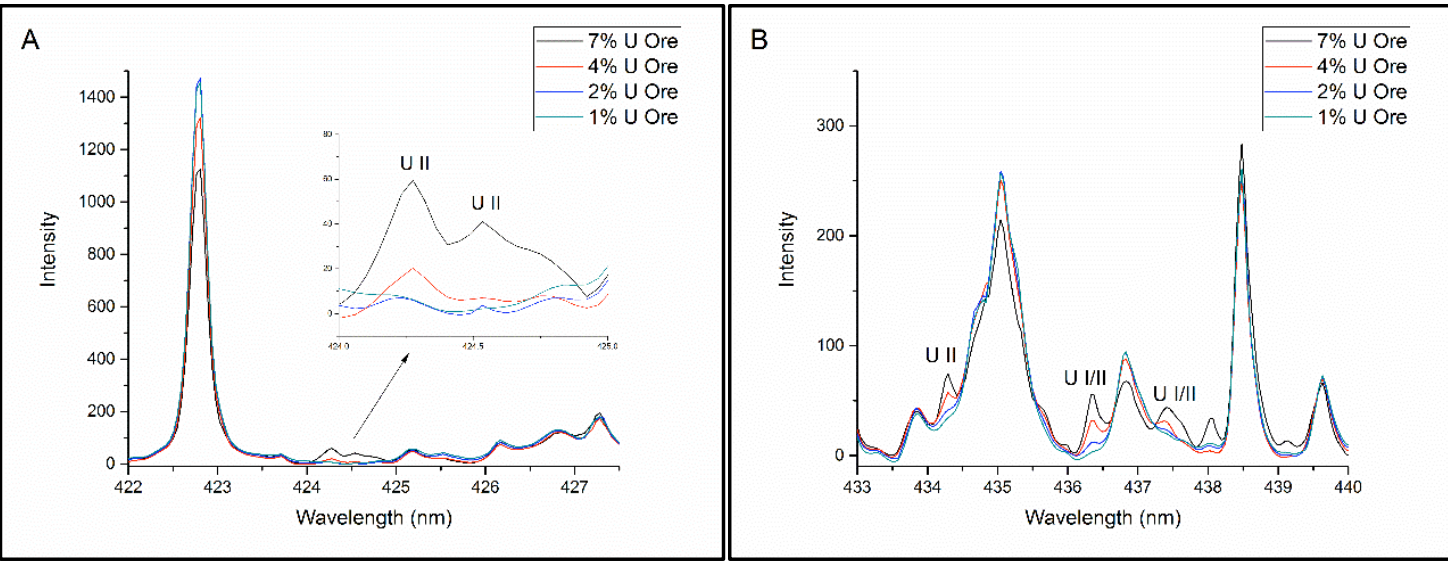

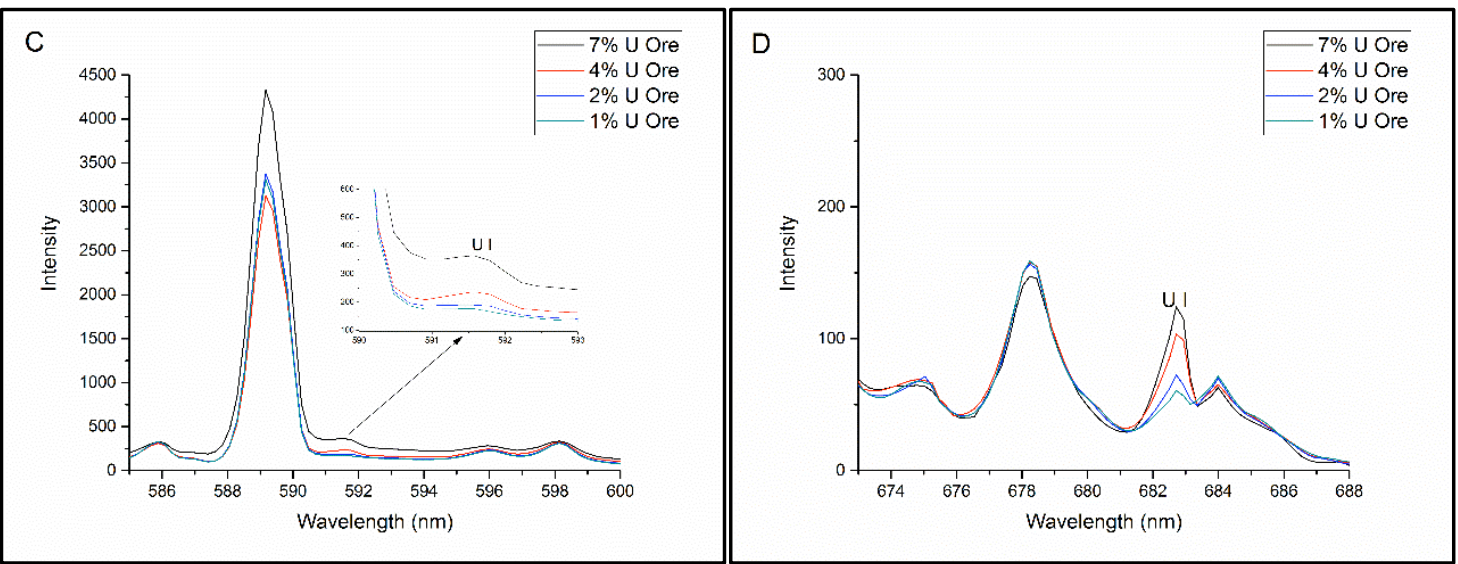

Fig. 2. Four ore and dunite samples containing varying concentrations of uranium (1-7\%) were analyzed using ChemCam test bed LIBS system. Several spectral windows are shown where there is visible concentration dependence for uranium in the ore and dunite matrices under Mars atmosphere conditions: A) $422-427.5 \mathrm{~nm}$, B) $433-440 \mathrm{~nm}$, C) $585-600 \mathrm{~nm}$, and D) $673-688 \mathrm{~nm}$
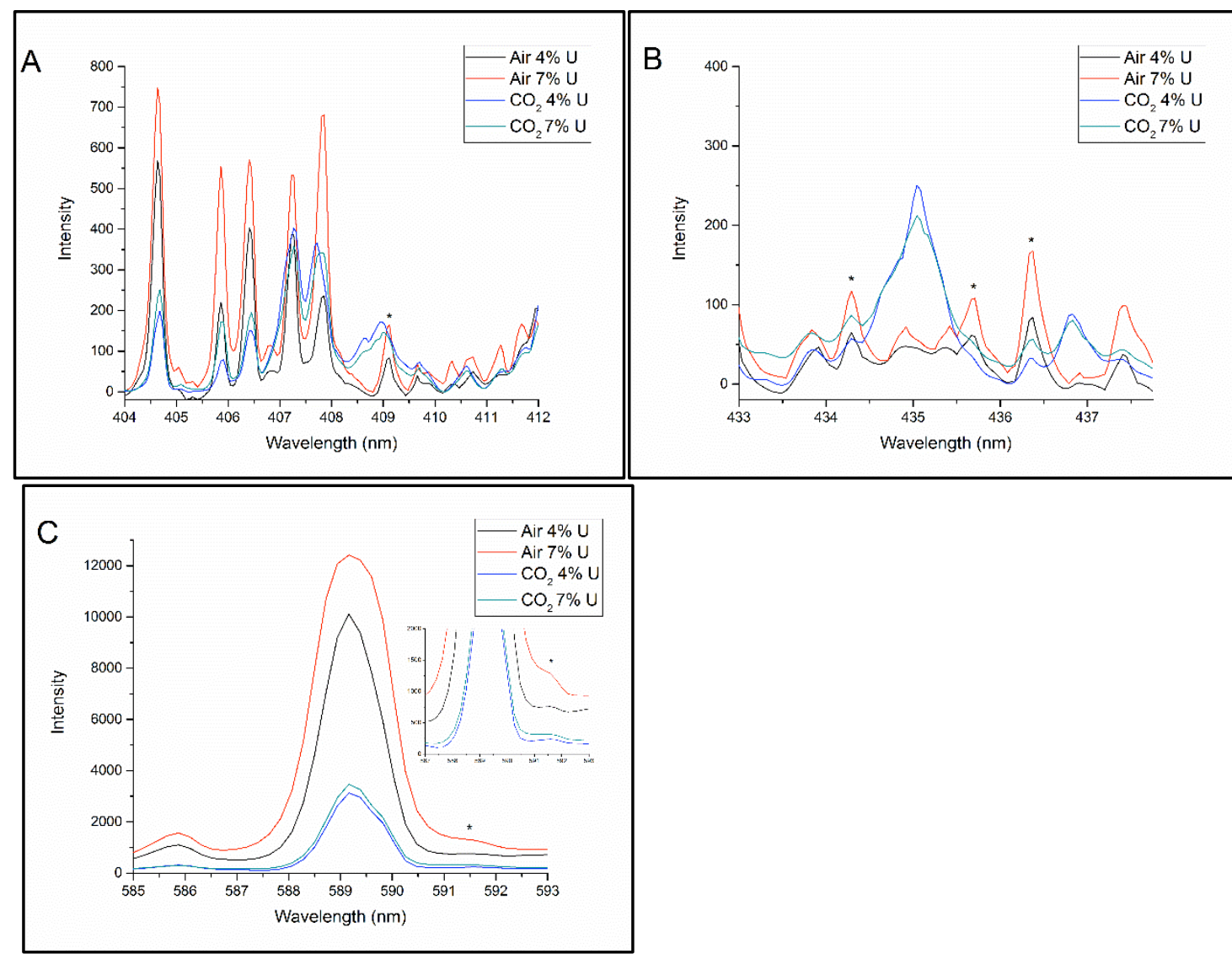

Fig. 3. Comparison of three LIBS spectral regions for standard atmospheric conditions and 7 Torr of $\mathrm{CO}_{2}$ for $4 \%$ and $7 \%$ uranium in dunite and ore: A) $404-412 \mathrm{~nm}$, B) $433-437.75 \mathrm{~nm}$, and C) $585-593 \mathrm{~nm}$ 
Table V Comparison of LOD of dunite in Air and $\mathrm{CO}_{2}$ atmosphere measured with the ChemCam test bed LIBS system.

\begin{tabular}{lcccc}
\hline & $\begin{array}{c}\text { Ambient Air LOD } \\
(\mathbf{p p m})\end{array}$ & $\mathbf{R}^{\mathbf{2}}$ & $\begin{array}{c}\text { 7 Torr } \mathbf{C O}_{\mathbf{2}} \mathbf{L O D} \\
(\mathbf{p p m})\end{array}$ & $\mathbf{R}^{\mathbf{2}}$ \\
\hline $\mathbf{4 0 9 . 0 1 3}$ U II & 24700 & 0.96 & interferent & \\
$\mathbf{4 2 4 . 1 6 7}$ U II & 23780 & 0.96 & $25760^{*}$ & 0.96 \\
$\mathbf{4 3 4 . 1 6 9}$ U II & 24390 & 0.96 & 40800 & 0.9 \\
$\mathbf{4 3 5 . 5 7 4}$ U I & 35880 & 0.92 & shoulder peak & \\
$\mathbf{4 3 6 . 2 0 5}$ U I & 19340 & 0.97 & 32050 & 0.93 \\
$\mathbf{5 9 1 . 5 3 9}$ U I & 47310 & 0.87 & 15340 & 0.98 \\
$\mathbf{6 8 2 . 6 9 2}$ U I & 18580 & 0.98 & 29080 & 0.95 \\
\hline
\end{tabular}

\section{In-Situ Ambient Medium Resolving Power Spectrometer Measurements on Uranium}

Uranium in an ore (7\% U, BL-5), $\mathrm{SiO}_{2}(0.01-1 \% \mathrm{U}, \mathrm{NBL} 102 \mathrm{~A}-105 \mathrm{~A})$, and a dunite $(0.5-4 \% \mathrm{U}, \mathrm{NBL}$ 42A series) matrix were analyzed using the in-situ medium resolving power spectrometer LIBS system under standard pressure and temperature to determine the effect matrix elements have on LOD's of uranium. The LIBS spectrum of uranium in an ore matrix (7\% U, BL-5) is shown in Figure 4A with an expanded spectral window shown in Figure 4B. In the expanded spectral window we assign atomic emission lines for uranium as well as other trace elements found in the ore (Ti, Fe, Ba, $\mathrm{Ca}, \mathrm{V}, \mathrm{Sr})$. Even using an echelle spectrometer with a resolving power of 10,000, there is considerable unresolved spectra that are a combination of background spectra and noise. Also, the spectral intensities of the matrix elements listed in Table II are stronger than the uranium atomic emission lines even though the concentration of uranium in the ore is higher. This is due in part to the fact that the excitation energy density available in the LIBS plasma must be spread out over the excited state distributions for all of the elements in the plasma. The high density of states for uranium and the actinide elements in general yield over 100,000 observable atomic emission transitions when the uranium atom is excited in a continuous wave (CW) or pulse laser generated plasma [26]. By contrast an element like iron yields a few thousand observable atomic emission transitions and silicon even less, as shown in Figure 5 A-B, respectively. The elements with the simplest electronic configurations compared to the actinide elements yield a lower density of states and therefore fewer but stronger atomic emission transitions even at lower concentrations. This leads to lower LODs for such elements. The main challenge in using LIBS to analyze actinide atoms in samples is to identify and assign atomic emission transitions that are as free from spectral complexity / overlap as possible with the highest degree of confidence. The identified atomic emission lines are then used to determine important sample properties for example concentration, composition, and elemental ratios.

To determine if a reduction in spectral complexity from the matrix (e.g., from dunite to silicon dioxide as shown in Figure 5) would yield lower limits of detection of uranium, two series of samples were analyzed: 1) uranium in a dunite matrix (0.05-4\% U, NBL 42A series) and 2) uranium in a silica matrix (0.01-1\% U, NBL 102A-105A). Figure 6A shows the $409 \mathrm{~nm}$ uranium emission line for the four different uranium samples in dunite: $0.5 \%, 1 \%, 2 \%$, and $4 \%$ uranium $(5,000 \mathrm{ppm}, 10,000 \mathrm{ppm}, 20,000 \mathrm{ppm}$, and $40,000 \mathrm{ppm}$, respectively). The uranium $409 \mathrm{~nm}$ emission line trends very well with concentration; therefore a calibration curve was generated to determine the LOD for this particular transition. The LOD was determined to be $6120 \mathrm{ppm}$ for uranium in a dunite (iron rich) matrix. Figure $6 \mathrm{~B}$ shows the $409 \mathrm{~nm}$ uranium emission line for the four different uranium concentrations in silica samples: $0.01 \%, 0.05 \%$, 
$0.1 \%$ and $1 \%$ uranium (100 ppm, $500 \mathrm{ppm}, 1,000 \mathrm{ppm}$, and 10,000 ppm, respectively). Again, the intensity of the uranium $409 \mathrm{~nm}$ emission line trends very well with concentration, although we note the somewhat unusual trend where the intensity of this line increases by a factor of only around 2 when the concentration increases from $0.1 \%$ to $1 \%$. The LOD for this transition is approximately $272 \mathrm{ppm}$ in the silica matrix.

The LOD found for uranium in silica samples compares very well to the lowest LOD reported for uranium in a nitric acid matrix / solution by Wachter and Cremers [7]. In the work reported by Wachter and Cremers, the matrix elements were hydrogen, nitrogen, and oxygen. These elements do not have complex electronic configurations and when excited in a LIBS type plasma yield simple spectral signatures with a few strong atomic emission transitions. In the case of silica, the matrix elements are silicon and oxygen. As previously discussed and shown in Figure 5B, the emission spectrum of $\mathrm{SiO}_{2}$ contains relatively few emission lines when compared to iron. The reduced spectral complexity as well as fewer excited states to share the plasma energy with results in lower LOD's for uranium in the $\mathrm{SiO}_{2}$ matrix. By contrast, a higher LOD of $6120 \mathrm{ppm}$ is obtained for the $409 \mathrm{~nm}$ transition in a dunite matrix, $(\mathrm{MgFe})_{2} \mathrm{SiO}_{4}$. Due to the increase in spectral complexity of the dunite matrix, the probability of spectral overlap increases. We also note that the spectral intensities of the uranium transitions are reduced significantly as well as the signal to noise in the presence of Fe. Table VI summarizes the LODs for the dunite and $\mathrm{SiO}_{2}$ matrices.

Table VI Summary of LODs determined using the echelle spectrometer $(10,000)$ LIBS system for uranium in dunite and $\mathrm{SiO}_{2}$ matrix.

\begin{tabular}{lcccc}
\hline & $\begin{array}{c}\text { Dunite Matrix } \\
\text { NBL 42A } \\
\text { Series LOD } \\
\text { (ppm) }\end{array}$ & $\mathbf{R}^{2}$ & $\begin{array}{c}\mathbf{S i O}_{\mathbf{2}} \text { Matrix } \\
\text { NBL } \\
\text { 101Series } \\
\text { LOD (ppm) }\end{array}$ & $\mathbf{R}^{2}$ \\
\hline $\mathbf{4 0 9 . 0 1 3}$ U II & 6120 & 0.99 & 272 & 0.97 \\
$\mathbf{4 2 4 . 1 6 7}$ U II & 5356 & 0.99 & 268 & 0.97 \\
$\mathbf{4 3 4 . 1 6 9}$ U II & 5693 & 0.99 & 402 & 0.95 \\
$\mathbf{4 3 5 . 5 7 4}$ U I & 6329 & 0.99 & 1067 & 0.83 \\
$\mathbf{4 3 6 . 2 0 5}$ U I & 2142 & 0.99 & 482 & 0.92 \\
$\mathbf{5 9 1 . 5 3 9}$ U I & gap & & gap & \\
$\mathbf{6 8 2 . 6 9 2}$ U I & 10741 & 0.97 & 720 & 0.91 \\
\hline
\end{tabular}




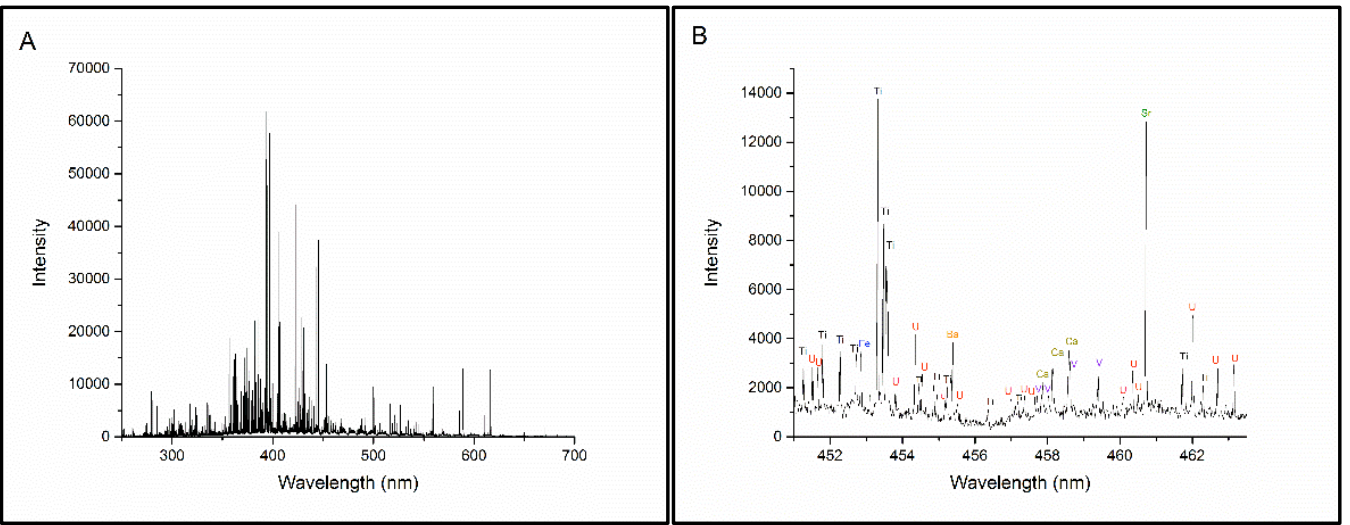

Fig. 4. The in-situ medium resolving power LIBS system was used to analyze uranium ore (BL-5). The overall spectrum can be seen in (A) 250-700 $\mathrm{nm}$ and an expanded and labeled spectral window can be seen in (B) 451-463 nm.

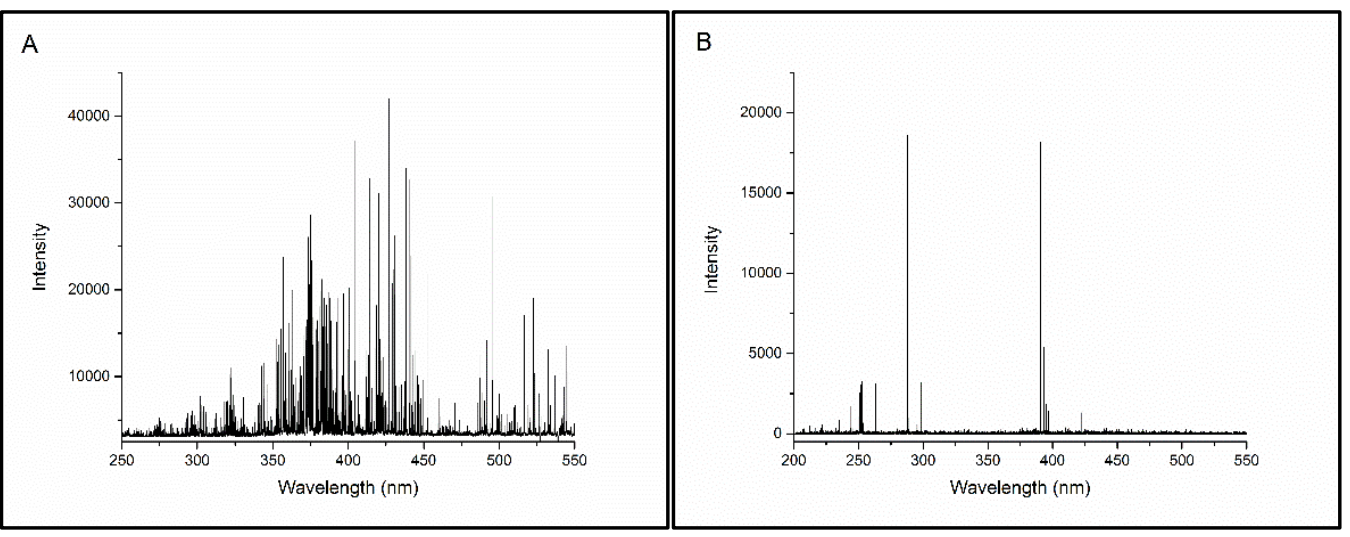

Fig. 5. LIBS spectra of (A) iron and (B) silicon dioxide showing the difference in spectral complexity represented by fewer emission lines in the silicon dioxide spectrum compared to iron.

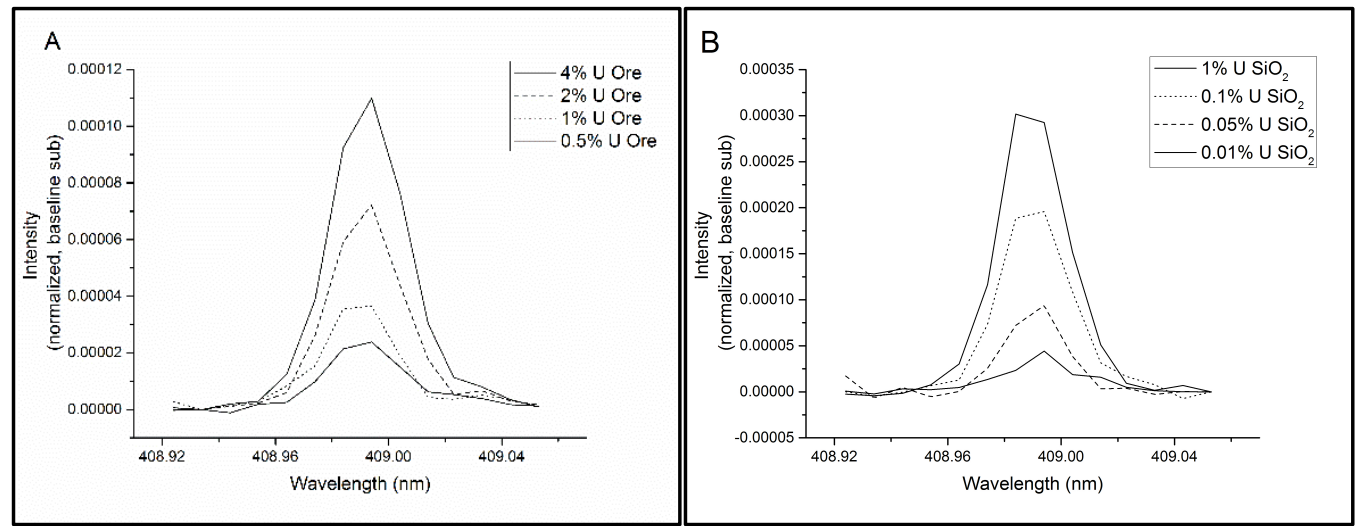

Fig. 6. (A) Concentration dependence for the $409 \mathrm{~nm}$ uranium emission line for the four uranium concentrations in dunite samples is shown. (B) Concentration dependence for the $409 \mathrm{~nm}$ uranium emission line for the four uranium concentrations in silica samples is shown. 


\section{Conclusions}

The importance of the measurement method (standoff vs. in-situ), matrix elements, and resolving power of a spectrometer on LOD is clearly shown in this work. With the ChemCam test bed LIBS system the LOD values range from 15336-40796 ppm in $\mathrm{CO}_{2}$ and $18585-47315 \mathrm{ppm}$ in air. While the LOD range for air is lower than in $\mathrm{CO}_{2}$, two of the peaks could not be determined (see Table V). The high LOD values are due to the resolving power and the standoff nature of the ChemCam test bed LIBS system measurements which as expected decrease as the number of photons collected by the optical system decreases by $1 / r^{2}$, where $r$ is the standoff distance. However, LOD results from the ChemCam test bed LIBS system are comparable with results of standoff measurements reported in the literature as previously discussed. The interferences at low pressure ( $<10$ Torr) in $\mathrm{CO}_{2}$ is consistent with literature where the LIBS spectra tend to degrade under these conditions due to lack of plasma confinement.

The role of matrix elements can be gleaned from the in-situ medium resolving power LIBS measurements. The LOD for uranium in the presence of a $\mathrm{SiO}_{2}$ matrix is $272 \mathrm{ppm}$ compared to 6,120 ppm in an iron-rich dunite matrix. There are several reasons for the large difference in LODs, including the finite energy in the plasma that must be shared with the increased number of excited states for iron in the dunite matrix. This results in lower intensities for the uranium atomic emission diagnostic transitions. Also, the spectral complexity and overlap is greatly reduced in the $\mathrm{SiO}_{2}$ matrix compared to the dunite matrix, allowing for higher $\mathrm{S} / \mathrm{N}$ for uranium to be detected. Even with the increase in the limit of detection due to the matrix effect (dunite matrix), the LOD's determined using the echelle spectrometer are 4 to 9 times lower than the corresponding values using the lower resolving power ChemCam LIBS system at standoff distances.

In conclusion, the detection and quantification of uranium varies significantly based on measurement distance, matrix elements present, atmosphere conditions, pressure and resolving power of the spectrometers used in the system. These measurements will assist one in selecting the proper system components based on the application and the required analytical performance.

\section{Acknowledgements}

The Los Alamos National Laboratory is operated by Los Alamos National Security, LLC for the NNSA of the U.S. DOE under Contract No. DE-AC5206NA25396. This work was carried out under Laboratory Directed Research and Development (LDRD) funding. The authors would also like to acknowledge DTRA for past support.

\section{References}

[1] D. A. Cremers and L. J. Radziemski, "Handbook of Laser-Induced Breakdown Spectroscopy", John Wiley \& Sons, Ltd., West Sussex, England, 2006. D. A. Cremers and L. J. Radziemski, "Handbook of Laser-Induced Breakdown Spectroscopy", (John Wiley and Sons, Ltd., West Sussex, England, $2^{\text {nd }}$ Edition,April 2013).

[2] A. W. Mizolek, V. Palleschi, and I. Schechter, "Laser-Induced Breakdown Spectroscopy (LIBS): Fundamentals and Applications", University Press, Cambridge, 2006.

[3] J. P. E. Singh and S. N. E. Thakur, "Laser Induced Breakdown Spectroscopy”, Elsevier B. V., Amsterdam, Netherlands, 2007.

[4] S. Musazzi and U. Perini, Editors, "Laser-Induced breakdown Spectroscopy”, (Springer Series in Optical Science, 2014).

[5] D. W. Hahn and N. Omenette, "Laser-Induced Breakdown Spectroscopy (LIBS)", part I: reviews of basic diagnostics and plasma-particle interactions: still-challenging issues within the analytical plasma community, Appl. Spectrosc. 64 (2010) 335A-366A.

[6] D. W. Hahn and N. Omenetto, "Laser-Induced Breakdown Spectroscopy (LIBS)", part II: review of instrumental and methodological approaches to material analysis and applications to different fields", Appl. Spectrosc. 66 (2012) 347-419. 
[7] J. Wachter and D. A. Cremers, "Determination of uranium in Solution Using Laser-Induced Breakdown Spectroscopy", Appl. Spectrosc. 1987. 41 (6):1042-1048.

[8] J. P. Singh, F. Y. Yueh, H. Zhang, K. P. Karney, "A Preliminary Study of the Determination of Uranium, Plutonium, and Neptunium by Laser-Induced Breakdown Spectroscopy”, Rec. Res. Dev. Appl. Spectrosc.1999.2:59-67.

[9] R. C. Chinni, D. A. Cremers, L. J. Radziemski, M. Bostian, C. Navarro-Northrup, "Detection of uranium Using LaserInduced Breakdown Spectroscopy”, Appl. Spectrosc. 2009. 63(11): 1238-1250.

[10] A. Sarkar, D. Alamelu, S. K. Aggarwal, Determination of trace consituents in thoria by laser induced breakdown spectrometry", J. Nuc. Materials, 384 (2009) 158-162.

[11] E. C. Jung, D. H. Lee, J. I. Yun, J. G. Kim, J. W. Yeon, K. Song, "Quantative Determination of Uranium and Europium in Glass matrix by Laser-Induced Breakdown Spectroscopy", Spectrochim. Acta B. 2011. 66 (9-10): 761-764.

[12] Y. S. Kim, B. Y. Han, H. S. Shin, H. D. Kim, E. C. Jung, J. H. Jung, and S. H. Na, "Determination of uranium in an ore sample using laser-induced breakdown spectroscopy", Spectrochim. Acta Part B 74-75 (2012) 190-193.

[13] I. Choi, G. C.-Y. Chan, X. Mao, D.L. Perry, R.E. Russo, Line Selection and Parameter Optimization for Trace Analysis of Uranium in Glass Matrices by Laser-Induced Breakdown Spectroscopy (LIBS), Appl. Spectrosc. 2013. 67(11): $1275-1284$.

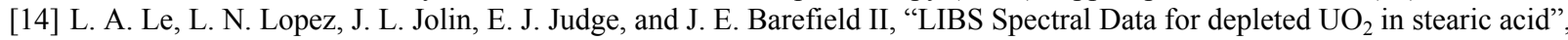
Los Alamos National laboratory Unclassified Report LA-UR 11-05566, Los Alamos National Laboratory, Los Alamos, N M. 2011, pp 153.

[15] E. J. Judge, L. A. Le, L. N. Lopez, J. L. Jolin, and J. E. Barefield II, "LIBS Spectral Data for Uranium Ore”, Los Alamos National laboratory Unclassified Report LA-UR 11-07095, Los Alamos National Laboratory, Los Alamos, N M. 2011, pp 249.

[16] E. J. Judge, L. A. Le, L. N. Lopez, J. L. Jolin, and J. E. Barefield II, "LIBS Spectral Data for Thorium Oxide in Stearic acid", Los Alamos National laboratory Unclassified Report LA-UR 11-07097, Los Alamos National Laboratory, Los Alamos, N M. 2011, pp 144.

[17] E. J. Judge, L. A. Le, L. N. Lopez, J. L. Jolin, and J. E. Barefield II, "LIBS Spectral Data for a Mixed Actinide Fuel Pellet Containing Uranium, Plutonium, Neptinum, and Americium”, Los Alamos National laboratory Unclassified Report LA-UR 12-22287, Los Alamos National Laboratory, Los Alamos, N M. 2011, pp 144.

[18] E. J. Judge, J. E. Barefield II, J. M. Berg, S. M. Clegg, G. J. Havrilla, V. M. Montoya, L. A. Le, and L. N. Lopez, "LaserInduced Breakdown Spectroscopy measurements of uranium and thorium powders and uranium ore", Spectrochim. Acta Part B 83-84 (2011) 28-36.

[19] J. E. Barefield II, E. J. Judge, J. M. Berg, S. P. Willson, L. A. Le, and L. N. Lopez, “Analysis and Spectral Assignments of Mixed Actinide Oxide Samples Using Laser-Induced Breakdown Spectroscopy(LIBS)”, Appl. Spectrosc. 2012.67 (4): $433-$ 440.

[20] R.C. Wiens, S. Maurice, J. Lasue, O. Forni, R.B. Anderson, S. Clegg, S., Bender, D. Blaney, B.L. Barraclough, A. Cousin, L. Deores, D. Delapp, M.D. Dyar, C. Fabre, O. Gasnault, N. Lanza, J. Mazoyer, N. Melikechi, P.-Y. Meslin, H. Newsom, A. Ollila, R. Perez, R.L. Tokar, D. Vaniman, Pre-Flight calibration and initial data processing for the ChemCam Laser-Induced Breakdown Spectroscopy instrument on the Mars Science Laboratory Rover, Spectrochimica Acta B 82 (2013)1-27.

[21] D. A. Cremers, A. Baddingfield, R. Smithwick, R. C. Chinni, C. R. Jones, B. Beardsley, and L. Karch,Appl. Spectrosc., 2012 (March), 66(3):250-261.

[22] M.E. Swartz, I.S. Krull, Handbook of Analytical Validation, Boca Raton FL, CRC Press 2012.

[23] Lasue J., Wiens R.C., Clegg S.M., Vaniman D.T., Joy K.H., Humphries S., Mezzacappa A., Melikechi N., McInroy R.E., and Bender S. (2012) Laser induced breakdown spectroscopy (LIBS) for lunar exploration. J. Geophys. Res. Planets, 117, E01002, doi :10.1029/2011JE003898.

[24] S. Maurice, R.C.Wiens, M. Saccoccio, B. Barraclough, O. Gasnault, O. Forni, N. Mangold, D. Baratoux, S. Bender, G. Berger, J. Bernardin, M. Berthé,N. Bridges, D. Blaney, M. Bouyé, P. Caïs, B. Clark, S. Clegg, A. Cousin, D. Cremers, A. Cros, L. DeFlores, C. Derycke, B. Dingler, G. Dromart, B. Dubois, M. Dupieux, E. Durand, L. d'Uston, C. Fabre, B. Faure, A. Gaboriaud, T. Gharsa, K. Herkenhoff, E. Kan, L. Kirkland, D. Kouach, J.-L. Lacour, Y. Langevin, J. Lasue, S. Le Mouélic, M. Lescure, E. Lewin, D. Limonadi, G. Manhès, P. Mauchien, C. McKay, P.-Y. Meslin, Y. Michel, E. Miller, H.E. Newsom, G. Orttner, A. Paillet, L. Parès, Y. Parot, R. Pérez, P. Pinet, F. Poitrasson, B. Quertier, B. Sallé, C. Sotin, V. Sautter, H. Séran, J.J. Simmonds, J.-B. Sirven, R. Stiglich, N. Striebig, J.-J. Thocaven, M.J. Toplis, D. Vaniman, The ChemCam Instrument Suite on the Mars Science Laboratory (MSL) Rover: Science Objectives and Mast Unit Description. Space Sci Rev (2012) 170:95-166.

[25] Wiens R.C., Maurice S., Barraclough B., Saccoccio M., Barkley W.C, Bell J.F. III, Bender S., Bernardin J., Blaney D., Blank J., Bouye M., Bridges N., Cais P., Clanton R.C., Clark B., Clegg S., Cousin A., Cremers D., Cros A., DeFlores L., Delapp D., Dingler R., D’Uston C., Dyar M.D., Elliott T., Enemark D., Fabre C., Flores M., Forni O., Gasnault O., Hale T., Hays C., Herkenhoff K., Kan E., Kirkland L, Kouach D., Landis D., Langevin Y., Lanza N., LaRocca F., Lasue J., Latino J., Limonadi D., Lindensmith C., Little C., Mangold N., Manhes G., Mauchien P., McKay C., Miller E., Mooney J., Morris R.V., Morrison L., Nelson T., Newsom H., Ollila A., Ott M., Pares L., Perez R., Poitrasson F., Provost C., Reiter J.W., Roberts T., Romero F., Sautter V., Salazar S., Simmonds J.J., Stiglich R., Storms S., Streibig N., Thocaven J.-J., Trujillo T., Ulibarri M., Vaniman D., Warner N., Waterbury R., Whitaker R., Witt J., and Wong-Swanson B. (2012) The ChemCam Instruments on the Mars Science Laboratory (MSL) Rover: Body Unit and Combined System Performance. Spa. Sci. Rev. $170,167-227$, doi 10.1007/S11214-012-9902-4. 
[26] Byron A. Palmer, Richard A. Keller, and Rolf Engleman Jr, "An Atlas of Uranium Emission Intensities in a Hollow Cathode Discharge,” Los Alamos Scientific Laboratory report LA- 8251-MS (July 1980).

[27] R. L. Kurucz and B. Bell, 1995 Atomic Line Data, Kurucz CD-ROM No. 23 Cambridge, Mass

[28] Yu. Ralchenko, A. E. Kramida, J. Reader, and NIST ASD Team (2011). NIST Atomic Spectral Database (ver. 4.1.0), [Online]. Available:Http://physics.nist.gov/asd3 [2011, August 31]. National Institute of Standards and Technology, Gaithersburg, MD.

[29] A. J. Effenberger Jr. and Jill Scott, Sensors (Basel), 2010;10(5):4901-4925.

[30] P. Ko, K. C. Hartig, J. P. McNutt, R. B. D. Schur, T. W. Jacob-Hood, and I. Jovanovic, “Adaptive femtosecond laserinduced breakdown spectroscopy of uranium", Review of Scientific Instruments, 84, 013104(2013). P. K. Morgan, J. R. Scott, and I. Jovanovic, "Hybrid Interferometric/Dispersive Atomic Spectroscopy of Laser-Induced Uranium Plasma", Spectrochimica Acta Part B, 116(2016)58-62. 